\title{
Aproximación antropológica al síndrome de dolor miofascial. Una propuesta de intervención
}

\author{
J. Aceituno Gómez y y A. Muñoz González² \\ ${ }^{1}$ Servicio de Rehabilitación. Hospital General Nuestra Señora del Prado. Talavera de la Reina (Toledo) \\ ${ }^{2}$ Servicio de Urgencias. Hospital Virgen de la Salud. Toledo
}

Aceituno Gómez J, Muñoz González A. Aproximación antropológica al síndrome de dolor miofascial. Una propuesta de intervención. Rev Soc Esp Dolor 2013; 20(4): 170-175.

\section{ABSTRACT}

Objectives:

- Study the myofascial pain syndrome (MPS) from a different perspective to the clinical one.

- Approach chronic MPS by means of an interdisciplinary point of view.

- Develop a proposal for intervention in patients with chronic MPS considering anthropological aspects of pain.

Methods: Work has been divided into two parts: the first one in which you conduct a search of scientific literature related to the object of study and a second one in which, from the results obtained, conclusions are drawn up to propose intervention.

Results: Anthropological aspects of MPS have not been a regular subject of study. Therefore it has been necessary to use studies on pain in general:

- Pain goes beyond the personal and subjective experience of those who suffer, the expression, and validation by social environment respond to socio-cultural factors

- Acute and chronic pain have a different socio-cultural connotations, scientific literature indicates the importance of finding causality for a more positive experience of pain.

- The attitude of the therapist and patient information are key elements in the process of recovery.

Conclusions:

- Understand the pain not only as a natural and subjective fact but also as a socially learned and culturally transmitted behaviour, influenced by many external factors.
- Need to integrate empathy and "estrangement" in addressing the patient with MPS.

- Inform the patient clearly and concisely about his recovery process to become an active part of treatment.

- Need to develop lines of research so that the study of MPS interacts with social sciences in order to obtain a greater interdisciplinarity which results in a holistic conception of the patient.

Key works: Anthropology. Myofascial pain. Physical therapy.

\section{RESUMEN}

Objetivos:

- Estudiar el síndrome de dolor miofascial (SDM) desde una perspectiva distinta a la clínica.

- Abordar mediante un enfoque interdisciplinar el SDM crónico.

- Elaborar una propuesta de intervención sobre el paciente con SDM crónico considerando aspectos antropológicos del dolor.

Métodos: Se ha dividido el trabajo en dos partes; una primera en la que se ha realizado una búsqueda de literatura científica relacionada con el objeto de estudio y una segunda en la que, a partir de los resultados obtenidos, se han elaborado las conclusiones a modo de propuesta de intervención.

Resultados: Los aspectos antropológicos del SDM no han sido un objeto de estudio habitual por lo que ha sido necesario recurrir a los trabajos sobre el dolor en general:

- El dolor va más allá de la experiencia personal y subjetiva del que lo padece, la expresión del mismo y la validación por parte del entorno social responde a factores socioculturales.

- El dolor agudo y el dolor crónico tienen unas connotaciones socio-culturales distintas, la literatura científica señala la importancia de encontrar la causalidad para una vivencia más positiva del dolor.

- La actitud del terapeuta y la información al paciente son elementos claves en el proceso recuperador. 
Conclusiones:

- Entender el dolor no solo como un hecho natural y subjetivo sino también como una conducta socialmente aprehendida y culturalmente transmitida, influenciada por muchos factores externos.

- Necesidad de integrar empatía y "extrañamiento" en el abordaje del paciente con SDM.

- Informar al paciente de manera clara y concisa acerca de su proceso de recuperación para convertirle en parte activa del tratamiento.

- Necesidad de diseñar líneas de investigación que imbriquen al estudio del SDM con las ciencias sociales en aras a una mayor interdisciplinariedad que redunde en una concepción holística del paciente.

Palabras clave: Antropología. Dolor miofascial. Fisioterapia

\section{INTRODUCCIÓN}

El síndrome de dolor miofascial (SDM), por definición, es un trastorno no inflamatorio que se manifiesta por dolor localizado, rigidez y cuya característica primordial es la presencia de "puntos gatillo" (1).

El SDM es una causa muy frecuente de problemas músculo-esqueléticos y estos, a su vez, constituyen la causa más frecuente de dolor (Fig. 1) agudo y crónico, y discapacidad, temporal o permanente, y como cualquier otro síndrome doloroso debe ser objeto también de una observación bajo la mirada antropológica que nos permita definirlo y nos facilite su comprensión en tanto en cuanto como criterio diagnóstico y como guía terapéutica. Todo ello nos ayudará a comprender e intentar un mayor acercamiento a un síndrome que, aún con esa prevalencia descrita en numerosos estudios que lo cifran en un $30 \%$ de los pacientes atendidos en atención primaria (2), un $20 \%$ en las consultas de reumatología (3) y entre el 85 y $93 \%$ en las unidades de dolor $(4,5)$, sigue siendo una gran desconocida por numerosos profesionales sanitarios, debido, en parte, a la falta de conocimiento de la propia patología, la falta de preparación en la materia y la carencia de fisioterapeutas preparados en unidades de dolor en España.

El estudio del síndrome de dolor miofascial en su aspecto crónico debe ser tratado desde diferentes ópticas que lo aproximen hacia una visión multi e interdisciplinar que nos permitan comprender al ser humano, diana de nuestra atención en su totalidad. Para ello hemos realizado, basándonos en bibliografía previa, una aproximación antropológica hacia el paciente con síndrome de dolor miofascial que nos conduce a una propuesta metodológica para su mayor comprensión, de tal forma que pueda repercutir en una mejor atención hacia el enfermo y mayor preparación del profesional.

La incorporación de otras disciplinas como la antropología permitiría dar un nueva visión de este síndrome que favorecería nuevas hipótesis o nuevos puntos de vista que proporcionen una base científica holística con la inclusión y aportaciones que se puedan hacer desde la teoría social.

\section{MÉTODOS}

El presente trabajo se ha realizado desde dos perspectivas distintas y complementarias a la vez, en primer lugar se ha realizado una revisión de la bibliografía existente sobre la antropología del dolor miofascial, posteriormente se ha ampliado el horizonte de búsqueda eliminando el término miofascial.

En una segunda parte y a raíz de los textos bibliográficos considerados relevantes para el estudio, aportados por la teoría social y antropológica sobre el dolor, se ha elaborado una guía de aspectos antropológicos a tener en cuenta por el profesional sanitario a la hora de abordar un paciente con dolor miofascial.

La manera de desarrollar la presente comunicación se ha realizado siguiendo la forma de ensayo antropológico en donde se prima lo cualitativo frente a lo cuantitativo.

\section{RESULTADOS}

En primer lugar es interesante resaltar la escasez de literatura científica que aborda el SDM desde una óptica distinta a la eminentemente clínica y/o sanitaria. Desde la antropología social y cultural, el tema del dolor es abordado principalmente por las subdisciplinas de antropología del cuerpo y antropología médica, centrando sus investigaciones en el dolor crónico. En relación con estudios sobre trastornos músculo-esqueléticos la fibromialgia se convierten en una patología sobre la que inciden los investigadores

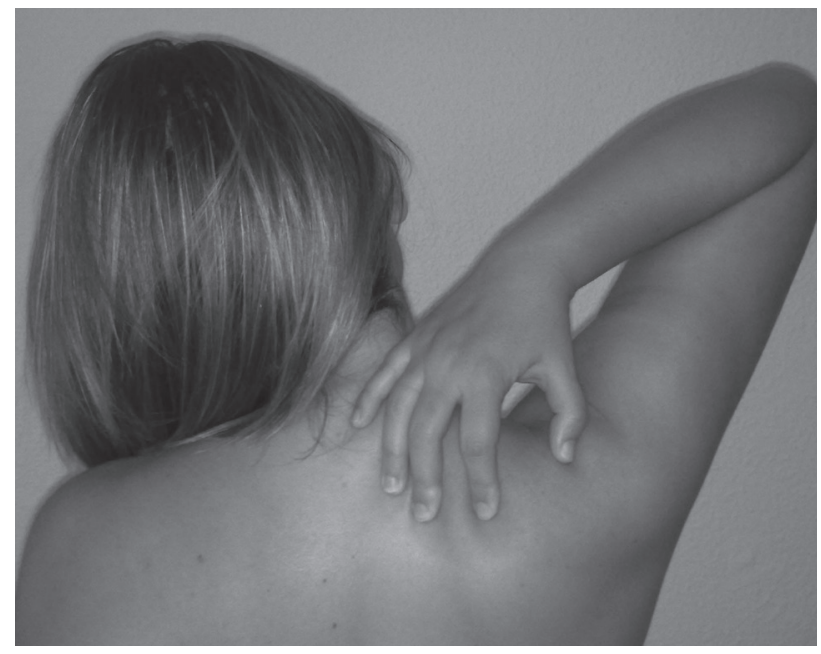

Fig. 1. Expresión de dolor. 
Rev. Soc. Esp. del Dolor, Vol. 20, N. o 4, Julio-Agosto 2013

sociales, trabajos como el de Tosal (6) en el que concluye sobre la importancia de la mediación del cuerpo en la relación sanitario-paciente en una consulta de reumatología a propósito de la fibromialgia, o el de Carrasco y Márquez (7) sobre el valor afectivo simbólico de los foros de fibromialgia; nos señalan esta patología como paradigmática del dolor crónico y establecedora de una visión holística en la que se imbrican aspectos separados por las divisiones más organicistas y separadoras de la tradicional medicina alopática, cuerpo y mente.

En el terreno antropológico, la enfermería, como una disciplina "cuidadora" y "curadora", nos lleva mucha ventaja a los profesionales de la terapia física como prolífica autora de artículos relacionados con la experiencia dolorosa, desde la cercanía de las disciplinas sanitarias, los textos de Barberá (8) y de Boixadera y Antón (9) nos dan claves que podemos importar al SDM.

La óptica del paciente con dolor crónico (perspectiva EMIC) es importante y necesaria por ser el contrapunto a nuestra visión sobre su proceso patológico (perspectiva ETIC) y tanto en la obra de Aullé (10) como en el artículo de Barragán-Solís (11) podemos observar la complejidad de dicha relación. Por todo ello, el objetivo inicial de una revisión sistemática de la bibliografía existente sobre la antropología del dolor miofascial ha experimentado una modificación que nos ha conducido a elaborar una propuesta, bajo la óptica antropológica, de elementos necesarios a tener en cuenta a la hora de tratar a un paciente con SDM.

Los resultados que hemos extraído de la bibliografía revisada los hemos agrupados bajo una serie de premisas o ítems que nos van a facilitar la elaboración de las conclusiones.

\section{El dolor tiene múltiples definiciones, dolor agudo versus dolor crónico}

La Asociación Internacional para el estudio del dolor (IASP en sus siglas en inglés), lo define como: "Una experiencia sensorial y emocional desagradable asociada con una lesión presente o potencial o descrita en términos de la misma".

Sin embargo, el Dr. Eduardo Ibarra (12), editorializa sobre la necesidad de una nueva definición del dolor categorizándolo como un "imperativo" de nuestros días, y propone la siguiente definición: "Una experiencia sensorial y emocional desagradable asociada con una lesión presente o potencial o descrita en términos de la misma y, si persiste, sin remedio disponible para alterar su causa o manifestaciones, una enfermedad por sí misma”.

Aquí establecemos un punto principal, la diferenciación entre dolor agudo y dolor crónico. Como profesionales sanitarios del ámbito público y debido a los modelos de derivación existentes es difícil el acceso a pacientes con SDM agudo, lo normal es que sean derivados pacientes con SDM crónico. Cuando se habla de dolor agudo, en el imaginario colectivo se entiende como algo transitorio, con una causa conocida que puede ser subsanada, según Boixadera y Antón (9) en el dolor agudo las quejas son culturalmente entendidas como lícitas, el problema es la gran laxitud e indefinición entre agudo y crónico para las ciencias de la salud y que en el marco puramente temporal se habla de 6 meses. En el dolor agudo, la presencia de una causa contingente, como puede ser por ejemplo, el caso de la activación del Trigger Point (TrP) 1 del sóleo en varones de mediana edad aficionados al senderismo de fin de semana por someterle a una tensión a la que no está acostumbrado; será entendido como transitorio, con una causalidad demostrada y con una intervención terapéutica que restablezca el nivel óptimo de salud de tal manera que resta el factor ansiedad de la ecuación dolorosa ya que es, en su planteamiento, un hecho finito.

El paciente que sufre SDM crónico, en muchas ocasiones mal diagnosticado y paliado con soluciones parciales y temporales (siguiendo con el ejemplo anterior: el problema surge en el diagnóstico, el desconocimiento de este tipo de síndrome, de los patrones de dolor referido, etc. hace que en el caso de que aparezca en la radiografía un espolón calcáneo, se descarte cualquier otro tipo de diagnóstico, tratando de manera conservadora o invasiva dicho espolón, sin que a largo plazo, cuando el sujeto inicie de nuevo su hobby reaparezca la sintomatología) siente el dolor como una carga, como un continuo "pedir permiso al cuerpo" para realizar actividad y una "constante explicación" para legitimar su padecimiento ante los demás debido a la ausencia de signos externos que sean percibidos como factor confirmante de lesión en el imaginario colectivo de nuestra sociedad.

Esta tendencia a la cronicidad de los pacientes con SDM se puede explicar desde dos vertientes clínicas:

- Respecto al diagnóstico: no existe una estandarización y accesibilidad de test específicos de laboratorio o estudios de imagen que permitan el diagnóstico del SDM de manera accesible para el clínico, siendo necesarios un exhaustivo examen físico y una minuciosa anamnesis e historia clínica del paciente, realizada por un profesional adiestrado en esta técnica y patología, ya que los estudios sobre evaluación de TrP determinan que hay una pobre fiabilidad entre los examinadores no entrenados y algo mejor en los entrenados $(13,14)$.

- Respecto al tratamiento: se basa en el diagnóstico, un mal diagnóstico conlleva un tratamiento erróneo y aumenta la tendencia a la cronicidad, incluso un buen diagnóstico no asegura un tratamiento óptimo sino resuelve el problema etiológico lo cual nos conduciría a la cronicidad. 


\section{El dolor como constructo social}

Le Breton (15) nos habla del dolor en dos direcciones que parecen antagónicas, pero que se implementan de manera necesaria e imprescindible para su comprensión; el dolor es íntimo, es subjetivo, solo puede ser sentido y experimentado por el propio sujeto; pero a la vez el dolor es fruto de la colectividad, de la sociedad en que se encuentra inserto el individuo, es educacional, cultural, social y relacional. El dolor es subjetivo, sin embargo la expresión del mismo es aprehendida por el sujeto a través de las experiencias y condicionantes que le brinda el entorno (Fig. 2). Boixadera y Antón (9) señalan que el individuo, conforme a los valores culturales que lo integran, toma conciencia de qué es considerado dolor y cómo se debe expresar dentro de su grupo de pares. Tanto en la obra de Le Breton (15), como en la conferencia de Silvia Balzano (16), se hacen referencias continuas, para enfatizar el carácter aprehendido social y culturalmente de la expresión del dolor, al estudio llevado a cabo por Mark Zborowski, (People in pain), en donde observó y analizó las formas de expresión del dolor de individuos pertenecientes a distintos orígenes, obteniendo distintos patrones de conducta expresiva y de relación con el médico y con el entorno, en base al origen y contexto socio-cultural del que son partícipes, si bien para Balzano (16) aunque parezca que refleja ciertos estereotipos, sí que se une al planteamiento de Zborowski de que existe una identidad cultural asociada al dolor, es decir, los comportamientos expresivos situados en una línea continua cuyos extremos serían por un lado: la absoluta falta de expresividad y en el contrario la dramatización del mismo, están condicionados por el entorno familiar y social que va moldeando la respuesta del sujeto; cuándo quejarse, cómo quejarse, etc. Barragán-Solís (11) concluye que la experiencia del dolor crónico se amplía desde el enfermo incluyendo al grupo familiar y al de relación, con lo que todo ello conlleva. En esta línea profundizan Boixadera y Antón (9) cuando señalan que el dolor, a pesar de ser un hecho íntimo, se encuentra impregnado de materia social, cultural, relacional y a la vez fruto de una educación. Según Steihaug y Malterud (17) aludiendo a la perspectiva fenomenológica enclavan el cuerpo humano como en constante reacción y formación a través de la relación con el entorno.

\section{La importancia de la información previa}

En este sentido Le Breton (15) nos muestra los resultados de un estudio del año 1964 en el que se observa claramente la importancia de una información previa exhaustiva y adaptada, no solo acerca de la intervención y de los resultados sino también del proceso postoperatorio y de recuperación posterior, en donde el paciente ante el conocimiento de los hechos que van a acaecer los tiene identificados. En palabras textuales de la obra Antropología del dolor "Un dolor identificado con una causa tiene un significado, es más soportable que un dolor que permanece en el sin sentido, no diagnosticado, incomprendido por el sujeto" En este momento nuestro trabajo como fisioterapeutas se tiene que centrar en informar y explicar todo aquello que le ha sido vetado previamente, a la vez que la actuación con objetivos a corto plazo para que recupere la confianza en el proceso de recuperación en base a discretos logros personales diarios. La eficacia, como señala Le Breton (15) en un estudio acerca de un procedimiento quirúrgico para el angor pectoris, depende en parte de la convicción que comparten el personal sanitario y el enfermo acerca de la utilidad del tratamiento. La subjetividad del dolor, su imbricación con la dimensión simbólica hace que pacientes tratados de una manera profesional y técnica correcta varíen en su proceso de mejora y disminución de sintomatología con la variable dependiente de la empatía del terapeuta que se hace cargo de su caso. No obstante, a la hora del abordaje del paciente con SDM el hecho de informar al paciente no se debe confundir con una inducción al dolor referido, el paciente debe expresar su dolor y nosotros interpretarlo sin direccionarle hacia uno o varios de los patrones desarrollados por Travell y Simons.

Ese "dar nombre al dolor" es de crucial importancia para los pacientes que nos llegan derivados tras un largo peregrinaje por las consultas más variopintas; y este hecho nos abre al siguiente ítem.

\section{Entender el dolor, la importancia del "extrañamiento profesional"}

A propósito de este caso, pero extensible a toda la práctica cotidiana, para la valoración del dolor nos vemos obligados a recurrir a unos conceptos muy conocidos en la antropología: la perspectiva EMIC (la que nos ofrece el paciente, su propia auto-percepción) y la perspectiva ETIC (la del profesional). A menudo ambas no coinciden.

Relativo a la implicación de los aspectos culturales en la expresión del dolor Boixadera y Antón (9) advierten de los posibles conflictos que pueden surgir cuando el terapeuta (en su caso hablan de enfermería) y el paciente no comparten los mismos valores culturales; falta de entendimiento, extrañeza y desconfianza son algunos de ellos y pueden causar, si no se tienen en cuenta, interferencias en el tratamiento y la evolución.

En algunos casos ese cuerpo sufriente y doliente el paciente lo deja en manos del profesional, consulta lo que puede y no puede hacer, lo responsabiliza de los éxitos y lo culpabiliza de los fracasos, expresiones como "que me has hecho" o "tienes manos de santo", ilustran estos casos en los que el profesional debe establecer una distancia con 


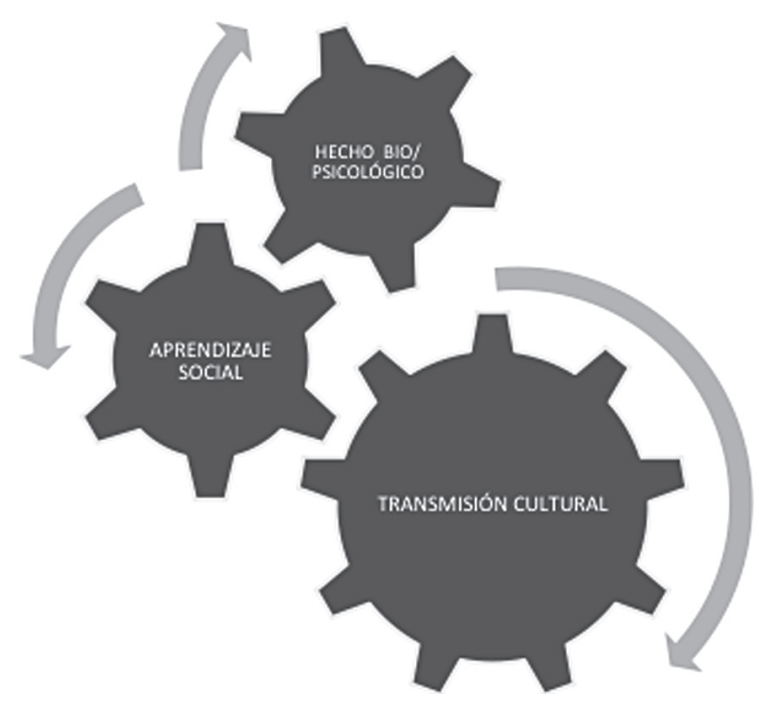

Fig. 2. Algunos factores involucrados en la expresión del dolor.

el sufrimiento del paciente para poder llevar a cabo los tratamientos con las máximas garantías, pero este "extrañamiento" del paciente no debe trasformarse en una frialdad de trato, una descortesía, una falta total de empatía y una desmotivación en el trabajo, todas estas actitudes, como bien nos cuenta Marta Allué (10), son percibidas desde lejos por los pacientes y ellos que nos confían su "cuerpo" hablando física y psicológicamente, deben ser receptores de algo más que unos cuidados técnicos, deben considerarse en ese momento, en el que son tratados, que son el centro de todos nuestros sentidos. Que su "cuerpo cedido" va a ser cuidadosamente tratado.

\section{CONCLUSIONES}

Basado en los resultados anteriores hemos elaborado una serie de pautas, desde el punto de vista antropológico, que sería interesante tener en cuenta a la hora de enfrentarnos con un paciente aquejado de dolor miofascial.

- Entender el dolor y por extensión su expresión no como un hecho natural sino como una conducta socialmente aprehendida y culturalmente transmitida, influenciada por muchos factores externos.

- Necesidad de integrar empatía y extrañamiento en el abordaje del paciente con dolor crónico, estableciendo una cuidadosa mezcla de ambos conceptos, especialmente si no están lo suficientemente diagnosticados.

- Informar de manera clara y concisa al paciente sobre nuestras actuaciones a la hora del diagnóstico y el tratamiento así como de la importancia del cumplimiento de las prescripciones realizadas.
- La manera de abordar el diagnóstico de un paciente con SDM basándonos en los patrones de dolor referido de los Trigger points ( $\mathrm{TrP}$ ) nos conduce a realizar sobre el paciente un proceso propio de la disciplina antropológica, la observación participante, recoger datos del paciente mediante lo que dice y lo que hace (algunas veces pueden no concordar) sin que medie por nuestra parte una inducción hacia su manera de sentir el dolor (como podría ser direccionarle hacia un determinado patrón de dolor referido). Informar sin dirigir.

- Importancia de diseñar líneas de investigación que imbriquen al estudio del dolor miofascial con las ciencias sociales en aras a una mayor interdisciplinariedad que redunde en una concepción holística del paciente.

\section{Agradecimientos}

Al Dr. Juan Antonio Flores (UCLM) por su tiempo y sus consejos. A la International Myopain Society por brindarnos la oportunidad y la excusa para elaborar este trabajo.

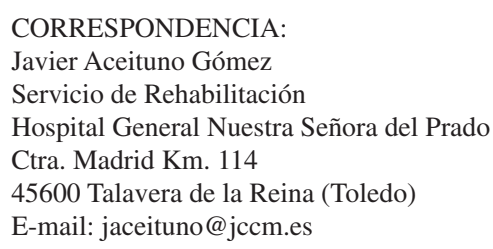

\section{BIBLIOGRAFÍA}

1. Travell J, Simons D, Simons L. Myofascial pain and dysfunction. In: Travell \& Simon (editors). The trigger manual. 2 ed. Baltimore: Williams \& Wilkins. 1999.

2. Skootsky S, Jaeger B, Oye RK. Prevalence of myofascial pain in general internal medicine practice. West J Med. 1989; 151:157-60.

3. Vanhoof J, Declerck K, Geusens P. Prevalence of rheumatic diseases in a rheumatological outpatient practice. Ann Rheum Dis. 2002;61:453-5.

4. Gerwin RD. Astudy of 96 subjects examined both for fibromyalgia and myofascial pain. J Musculos Pain. 1993;3:121.

5. Fishbain DA, Goldberg M, Meagher BR, et al. Male and female chronic pain patients categorized by DSM-III psychiatric diagnostic criteria. Pain. 1986;26:181-97.

6. Tosal B. El cuerpo como excusa. El diagnóstico de la fibromialgia en una consulta de reumatología. Index de Enfermería v.17 n.1 Granada enero-marzo 2008.

7. Carrasco M, Márquez M. El valor afectivo simbólico de los foros de fibromialgia \{Ponencia\} en XI Congreso de Antropología: retos teóricos y nuevas prácticas. Donostia, septiembre 2008.

8. Barbera F. Una visión de la antropología filosófica de enfermería: la empatía como cuidado trascendental. En: www. portalesmedicos.com. 
9. Boixadera, Antón. Aproximación al hecho del dolor desde la antropología cultural: implicaciones para la práctica enfermera. Ágora de Enfermería Digital. N 16. Barcelona. 2008.

10. Aullé M. Perder la piel. Una trágica experiencia y una heroica recuperación. Ed. Seix Barral. $7^{\mathrm{a}}$ ed. Barcelona. 2004.

11. Barragán-Solís A. El dolor crónico: una visión antropológica de acuerdo a familiares y pacientes. Archivos en medicina familiar. 2006;8(2):109-17.

12. Ibarra E. Una nueva definición de dolor. Un imperativo en nuestros días. Rev Soc Esp Dolor. 2006;13(2):65-72

13. Tough EA, White AR, Richards S, et al. Variability of criteria used to diagnose miofascial trigger point pain syndrome. Evidence from a review of the literature. Clin J Pain. 2007;23:278-86

14. Hsieh CY, Hong CZ, Adams AH, et al. Interexaminer reliability of the palpation of trigger points in the trunk and lower limb muscles. Arch Phys Med Rehabil. 2000;81:258-64.
15. Le Breton D. Antropología del dolor. Ed. Seix Barral. Barcelona. 1990.

16. Balzano Silvia. Homo dolens: hacia una antropología del dolor. En: http://www.lataccs.com.ar/.

17. Steihaug S, Malterud K. Stories about bodies: A narrative study on self-understanding and chronic pain. Scan J Prim Health Care, 2008;26:188-92.

18. Le Breton, D. Lo inaprensible del cuerpo. Antropología del cuerpo y modernidad. Nueva Visión. Buenos Aires. 1995. p. 13-27.

19. Otegui, R. Factores socioculturales del dolor y el sufrimiento En: Perdiguero E, Comelles JM (editores). Medicina y cultura. Estudios entre la antropología y la medicina. Ed Bellatera, Barcelona. 2000. p. 227-48.

20. Pain New essays on its nature and the methodology of its study. Edited by Murat Aydede 2005 Massachusetts Institute of technology. A Bradford Book The MIT Press. 